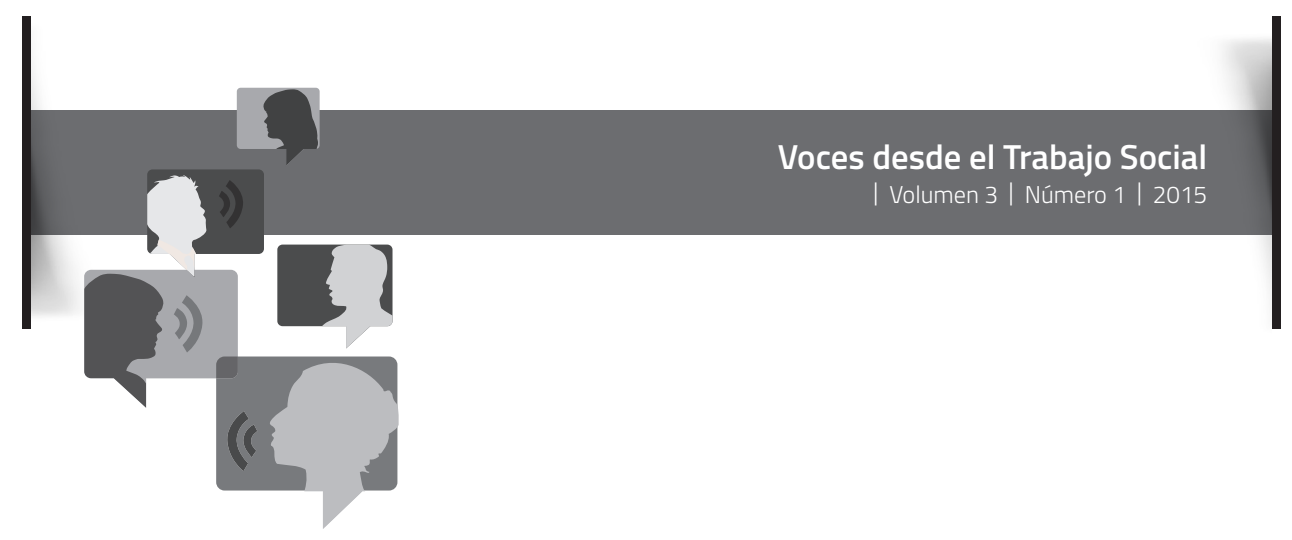

\title{
ALGUNOS ASPECTOS A CONSIDERAR AL INTERVENIR CON AGRESORES Y AGRESORAS DE VIOLENCIA DOMÉSTICA
}




\section{Gloria M. Molina Castro}

Catedrática Retirada de la Escuela Graduada de Trabajo Social Beatriz Lassalle de la Universidad de Puerto Rico. Directora Ejecutiva y Trabajadora Social Clínica del Institute for Individual, Group and Organizational Development, Inc., Programa AFANA de Gurabo. Adiestradora e Investigadora.

\section{Antonio Fernández}

Trabajador Social Clínico del Institute for Individual, Group and Organizational Development, Inc., Programa AFANA. Ha ofrecido cursos en la Universidad del Este en Puerto Rico. Adiestrador en temas relacionados con la violencia doméstica. Recibió el Premio Celestina Zalduondo en 2007.

\section{Yarisel Berríos}

Trabajadora Social Clínica del Institute for Individual, Group and Organizational Development, Inc., Programa AFANA. Supervisora de Instrucción Práctica de Trabajo Social de la Universidad Interamericana de Puerto Rico, Recinto Metropolitano. Adiestradora e Investigadora en temas relacionados con la violencia doméstica.

\section{Recibido:}

13 de febrero de 2015

\section{Aprobado:}

19 de septiembre de 2015 


\section{ALGUNOS ASPECTOS A CONSIDERAR AL INTERVENIR CON AGRESORES Y AGRESORAS DE VIOLENCIA DOMÉSTICA}

escrito por of

Gloria M. Molina Castro, Antonio Fernández

y Yarisel Berríos

\section{Resumen}

La violencia doméstica es uno de los graves problemas que impacta la sociedad puertorriqueña. El Proyecto de Resocialización Masculina y Femenina se ha dado a la tarea de intervenir con los agresores y las agresoras a nivel de prevención primaria y secundaria. A tales efectos, se presentan y se describen las etapas de cambio que experimenta el agresor y la agresora de violencia doméstica a través del proceso de intervención grupal e individual. Incluso, se describe una guía básica usada en el proceso de intervención con las personas agresoras de violencia doméstica, especificando las áreas que requieren cambios y algunas estrategias y técnicas de intervención. Por último, se presentan comentarios que expresan los agresores que reflejan el mensaje implícito de violencia contra la mujer y se ofrecen ideas de cómo cambiar dichos mensajes. 
Descriptores: violencia doméstica, agresores de violencia doméstica, metodología grupal, intervención profesional, relaciones de pareja.

\section{Abstract}

Domestic violence is one of the major problems impacting the Puerto Rican society. The Proyecto de Resocialización Masculina y Femenina has been working with male and female aggressors at the primary and secondary prevention level. To this end, we present the stages of change experienced by the aggressors of domestic violence through the process of group and individual intervention. A basic guide used in the intervention process is described, specifying the areas requiring changes and some strategies and techniques utilized in the program. Finally, participants' comments, expressing the aggressors' implicit message of violence against women are presented. Suggestions about how to change these messages are offered.

Keywords: domestic violence, domestic violence aggressors, group methodology, professional intervention, marital relations. 


\section{Introducción}

El Institute for Individual, Group and Organizational Development, Inc. (Instituto IGODI) es una organización sin fines de lucro con sede en la ciudad de Gurabo, Puerto Rico, que por espacio de diez años, a través de su Programa de Apoyo a Niños y Adultos (Programa AFANA), ha ofrecido intervención profesional de servicios socio-educativos a personas agresoras de violencia doméstica. A tales efectos, se desarrolló el Proyecto de Resocialización Masculina y Femenina (Proyecto PRMF), el cual es auspiciado por la Administración de Familias y Niños del Departamento de la Familia.

Los servicios en el Proyecto PRMF se ofrecen desde una perspectiva de prevención primaria y secundaria. Lo que se pretende en la prevención primaria es fortalecer y preparar a todas las personas para que se puedan enfrentar a cualquier situación que les pudiera impactar a lo largo de su vida (Molina, 2006). "Es como poner una vacuna social, desarrollando en todos los individuos auto-percepciones saludables, destrezas sociales, concienciándolos e identificando recursos de apoyo" (Pransky, 2003, p. 3). Esto se logra a través de talleres especializados en prevención de violencia en las relaciones de noviazgo, que se desarrollan en las escuelas de la comunidad (de 10 sesiones). Por otro lado, la prevención secundaria está dirigida a aquellas personas o grupos en los cuales se han identificado factores de riesgo o que están atravesando por una situación de crisis y están muy vulnerables en un estado temporal de trastorno y desorganización. El propósito de este nivel de prevención secundaria es realizar una intervención temprana con el "problema" o necesidad o la crisis para detener su desarrollo y posibles consecuencias de deterioro (Molina, 2006). Esto se logra en el Proyecto PRMF a través de los grupos socioeducativos y de resocialización. 
Cabe destacar que el Instituto IGODI, Programa AFANA, llevó a cabo un estudio longitudinal con 82 personas en el cual se midió la efectividad de los servicios ofrecidos a los agresores y a las agresoras desde el 2004 hasta el 2014 (Molina, Fernández y Berríos, 2015). Las variables que se tomaron en consideración y los resultados después de recibir los servicios, fueron: el 95\% de las personas agresoras no había tenido alguna orden de protección; el 99\% no había sido intervenido por el Departamento de la Familia por una situación de violencia doméstica; y el $96 \%$ no ha tenido incidentes de violencia doméstica. Estudios como este y el realizado por Flavia Arrigoni, José Luis Jiménez, José I. Navarro y Pedro Mendoza (2013) evidencian la efectividad y eficiencia de los programas y proyectos dirigidos a esta población y demuestran que las personas agresoras pueden lograr cambios cognitivos y conductuales luego de los procesos de intervención.

\section{Proceso de intervención}

Desde el año 2004 hasta septiembre del 2015 se han atendido 630 personas agresoras (participantes): 528 hombres y 102 mujeres. En sus comienzos, el Proyecto PRMF inició ofreciendo servicios a hombres agresores, pero en la marcha se comenzaron a recibir referidos de mujeres calificadas como agresoras por los profesionales de la conducta humana. Para ofrecer los servicios se utiliza la metodología grupal. Esto es así, ya que se puede lograr una práctica profesional mucho más efectiva con la utilización de la metodología grupal (Molina, 2006). El ambiente grupal ofrece una poderosa y única oportunidad de crecimiento y cambio. Las personas agresoras establecen una conexión y aprenden unos de otros nuevas conductas para lidiar con sus relaciones de pareja. Esto 
lleva al aprendizaje vicario, en el cual las personas integrantes del grupo aprenden de las experiencias de los demás y el proceso grupal puede facilitar la toma de decisiones por las experiencias previas de otros participantes (Bandura, 1986). Además, con esta metodología, el profesional de trabajo social tiene la oportunidad de utilizar una diversidad de técnicas que propician la dinámica grupal (Molina, 2006).

Las personas agresoras del Proyecto PRMF acuden a este por diferentes motivos, pero ninguno ha venido simplemente porque reconoció la existencia de una conducta violenta en la relación de pareja. La mayoría de las personas agresoras en el Proyecto PRMF acuden al proceso de ayuda de forma involuntaria, lo cual implica trabajar con la negación y la resistencia. Se puede definir como participante involuntario a aquella persona que recibe los servicios pero que no los buscó por cuenta propia. Existen dos tipos de participantes involuntarios: a) participantes legalmente obligados (participan de Programas de Desvíos a nivel de prevención terciaria, luego de ser encontrados culpables del delito según lo estipula la Ley \#54 del 1989, Ley para la Prevención e Intervención con la Violencia Doméstica); b) y participantes referidos por agencias o familiares (especialmente como condición de su pareja para continuar la relación). Jorge Corsi (2004) y David J. Decker (1999) en sus experiencias interviniendo con agresores, establecen que la "voluntariedad" de los agresores (hombre) al proceso de ayuda, en la mayoría de las situaciones, es condicionada por la esposa o compañera quien le ha advertido que si este no busca ayuda la relación culminará para siempre. Por estas circunstancias, la experiencia en el Proyecto PRMF, en muchas ocasiones representa la última oportunidad que tiene el agresor para continuar con su pareja. En la experiencia en el Proyecto PRMF se ha encontrado que, en algunas circunstancias, el agresor es asesorado por su abogado 
para que busque ayuda profesional, a fin de que cuando comparezcan a la vista preliminar en el Tribunal, se le presenta evidencia al Juez de que este participó de un programa de servicios (Molina \& Fernández, 2009).

Es necesario lidiar con la resistencia de la persona participante desde la entrevista de cernimiento. Por eso, con frecuencia, dicha entrevista no se puede reducir a 1 o 2 sesiones, pues se necesita el compromiso del participante con el Proyecto. Este compromiso se refiere a su fidelidad a la casuística, postulados y metodología que permean en el proceso de intervención. Mientras más positiva sea la percepción del sujeto hacia su rol participativo en el proceso de intervención, más probabilidades de éxito tendrá dicha intervención.

Detrás de cada una de las personas agresoras del Proyecto PRMF existen historias de vidas que reflejan los procesos de socialización, las conductas aprendidas y las experiencias de vida que dan pie a las ideas distorsionadas sobre roles de género y a conductas violentas (Ortiz \& García, 2004; Pérez, N., 2011; Molina \& Fernández, 2009). Por eso, el Proyecto PRMF pretende que el agresor y la agresora se sensibilicen de sus acciones mediante el autoanálisis de sus conductas. Además, que reconozcan que la violencia es una conducta aprendida y, por ende, puede ser desaprendida. Incluso, se espera que la persona analice profundamente las consecuencias de sus acciones violentas. Las personas violentas contra su pareja son responsables de sus conductas y, a su vez, tienen carencias importantes en el control de los impulsos, en sus sistemas de creencias, en los métodos que usan para comunicarse, en su de toma de decisiones, en sus destrezas de solución de problemas, en el control de los celos, en la percepción adecuada de sí mismo y en el conocimiento del proceso de construcción de género. Además de otras áreas de necesidad 
que podrían señalarse, es necesario resaltar que la inmensa mayoría de este colectivo, carece de un conocimiento mínimo sobre las leyes de prevención de la violencia doméstica y el maltrato de menores. Las áreas antes identificadas son algunas de las que se trabajan con las personas agresoras a través del proceso grupal en el Proyecto PRMF.

\section{Etapas de cambio en el proceso de intervención}

Durante el período en que se ha implantado el Proyecto PRMF, los trabajadores y trabajadoras sociales han recopilado una serie de experiencias en los procesos de intervención con esta población. A raíz del cúmulo de conocimientos y destrezas obtenidos en dicho proceso, se pretende compartir algunos aspectos que servirán de base para que otros profesionales continúen la labor iniciada por el Instituto IGODI, Programa AFANA. En primer lugar, a continuación se describen las etapas de cambio que experimenta el agresor y la agresora de violencia doméstica a través del proceso de intervención grupal e individual.

I. Negación: "Yo estoy bien, ella me provocó, yo soy la víctima". No reconoce que tiene un problema.

Tiende a minimizar el problema.

II. Coraje: "¿Quién me dice que soy un agresor o una agresora?"

III. Desplazamiento de la culpa: "La culpa es de otros, no mía". No asume responsabilidad por los episodios de violencia.

IV. Manejo de la resistencia al servicio: "Decido aceptar la ayuda". Aquellos que no manejan adecuadamente la resistencia, abandonan el servicio. 
V. Aceptación de la violencia doméstica como problema familiar: "Ahora sí entiendo que mis acciones tienen consecuencias". Muestra motivación necesaria para mantenerse en el proceso de ayuda y cumplir adecuadamente con las expectativas de la intervención.

VI. Aprendiendo nuevas estrategias de manejo: "Estoy aprendiendo a manejar mis frustraciones, coraje y otras más, como: toma de decisiones responsables, manejo del estrés, control de los celos y comunicación efectiva". Evidencia motivación para modificar su comportamiento agresivo.

VII. Aplicando estrategias nuevas a la relación de pareja: "Yo modifico conductas y tengo nuevas maneras de actuar, pensar y sentir". Gana sensibilidad en las necesidades físicas, afectivas y sociales de su pareja.

\section{Estrategias y técnicas de intervención para el cambio}

Como se ha señalado anteriormente, las personas agresivas contra su pareja, comúnmente evidencian carencias cognoscitivas, afectivas y conductuales significativas, tales como: distorsiones cognitivas, dificultades en las destrezas de comunicación, irritabilidad y falta de control de impulsos, entre otras; que requieren de una atención especial en los procesos de intervención profesional. A través de la experiencia en el Proyecto PRMF, se han identificado unas áreas, las cuales ameritan ser atendidas en el proceso de cambio que debe experimentar el participante durante la intervención social. Estas se describen en la Tabla 1. 


\section{Tabla 1:}

Guía básica en el proceso de intervención con agresores y agresoras de violencia doméstica:

\section{Aspecto a Cambiar}

Desconocimiento total de la Ley 54 del 1989 y las consecuencias de su aplicabilidad. Sensibilidad ante las necesidades sexuales de su pareja.

\section{Poca sensibilidad del impacto de la violencia en todos los miembros de la familia, en especial, en los hijos y las hijas.}

Pobre manejo del coraje, resultando en la ira.

\section{Estrategias y Técnicas}

Estudio y análisis de la ley: Remedio civil (orden de protección) y remedio criminal. Discusión de ejemplos concretos donde se aplica la ley. Películas o documentales.

Presentación de ejemplos reales del impacto de la violencia en la familia. Películas o documentales

Auto análisis de lo que produce el coraje y las reacciones más comunes al mismo.

Ejercicios prácticos para el manejo del coraje.

Pobre destrezas en la comunicación efectiva $y$ afectiva. para el desarrollo

\section{Ejercicios prácticos} de habilidades en la comunicación efectiva y afectiva. Películas cortas.

\section{Cambio Logrado}

Entendimiento de las consecuencias de violar la Ley 54.

Reconocer el impacto de la violencia en todos los miembros de la familia.

Control y manejo adecuado del coraje, identificando y aplicando estrategias de manejo. 


\section{Aspecto a Cambiar}

Deseos de su propia satisfacción sexual y poco entendimiento $y$ conocimiento de las necesidades sexuales de la pareja.

\section{Pobre destreza en la toma de decisiones responsables.}

Pobre manejo de otras emociones como: frustraciones, celos, etc.

Distorsión en las ideas sobre la construcción de género.

Desconocimiento de lo que es una relación de pareja saludable.

\section{Estrategias y Técnicas}

Educación sobre la sexualidad en la relación de pareja.

Educar sobre el proceso responsable de toma de decisiones y ejercicios prácticos al respecto.

Reflexión de situaciones en las cuales existen los celos y las consecuencias de estos y otras emociones mal manejadas.

\section{Educación sobre los} roles de género y la igualdad entre la mujer y el hombre.

Discusión y análisis de situaciones reales e hipotéticas de relaciones de pareja saludables y de relaciones no saludables.
Déficit en la solución de problema sin el uso de la violencia.

Discusión y análisis de situaciones reales e hipotéticas que representen la necesidad de solución de problema.

Percepción equivocada
de que su pareja le
pertenece.

Manejo de comentarios
de las personas
agresoras que reflejen
esta percepción
distorsionada.

\section{Cambio Logrado}

Sensibilidad ante las necesidades sexuales de su pareja.

\section{Toma de decisiones} responsablemente.

Reconocimiento de que los celos dañan la relación de pareja y en nada aportan para fortalecer la misma

\section{Reconocimiento y} aceptación de la igualdad de género.

Identificación de las características de una relación de pareja saludable.

Internalización de que cada acción tiene unas consecuencias, sean positivas o negativas.
Reconocer que su pareja no le pertenece y que no es de su propiedad. 


\section{Aspecto a Cambiar}

Pobre conciencia de que la infidelidad representa un daño emocional a su pareja.

\section{Estrategias \\ y Técnicas}

Discusión y análisis de literatura relacionada con el tema.

\section{Cambio}

Logrado

Fidelidad en la relación de pareja.

Es importante recalcar que, a través del proceso de intervención, surgen comentarios de las personas agresoras que reflejan claramente estas áreas y la perpetuidad de la violencia y el poder de uno sobre el otro. Estos comentarios se tienen que trabajar para que puedan lograr los cambios esperados. Es labor de los trabajadores y trabajadoras sociales detener la discusión de los temas y procesar estos comentarios que exteriorizan las personas agresoras. Se muestran a continuación algunos de los comentarios que han realizado las personas agresoras, en especial los hombres, en la experiencia grupal y con los cuales se ha trabajado con el fin de cambiar el mensaje implícito en dichos comentarios, (véase Tabla 2). Cabe destacar que esto es solo una muestra de los comentarios que se han escuchado y manejado a través de la intervención con esta población. 


\section{Tabla 2:}

\section{Discurso de los Hombres Agresores de Violencia Doméstica:}

\section{Comentarios}

"Yo a la mía le doy permiso para que salga por ahí".
"Simplemente le di un empujoncito y accidentalmente ella se cayó".
"Ella es una vaga, no trabaja ni hace nada en la casa".
"La celo porque la amo".

"Ella es una bruta, no entiende lo que le digo".

\section{Discurso \\ (el mensaje implícito en el comentario)}

Él controla las decisiones de ella. Ella es inferior a él, por lo tanto, es este el que da permiso como si fuera ella una menor. Sentido de propiedad, la mujer le pertenece al hombre.

El agresor minimiza su conducta violenta. Él no se siente responsable de su conducta.

No reconoce ni valoriza el trabajo que realiza la mujer en el hogar. Considera que las tareas del hogar son exclusivas de la mujer.

\section{Cambiando el Discurso (deconstrucción)}

Ella es adulta, puede tomar decisiones y ser responsable a las consecuencias de sus actos. La mujer no es una propiedad del hombre. Es indispensable mantener una relación de igual.

Cada persona es responsable de sus actos. Reconocer el impacto de su conducta a nivel de pareja y familiar.

\section{El trabajo no} remunerado en el hogar es mucho mayor que uno fuera de este, en términos de esfuerzo, tiempo y energías.

Las tareas del hogar no son exclusivas de uno u otro, sino que deben de ser compartidas.

Para amar hay que celar, es un requisito indispensable.

La mujer es inferior al hombre y como tal no tiene la capacidad de entender el mensaje.
El que verdaderamente ama, no cela porque en una relación saludable permea la confianza.

\section{El entendimiento y} el intelecto no son exclusivos del hombre o de la mujer. 


\section{Comentarios}

"Yo la ayudo con los nenes, ella no se puede quejar".

"Esa mujer tiene un demonio por dentro, nunca está de acuerdo con lo que le digo".

"Ella es la que me provoca y me hace perder el control".

\section{Discurso}

(el mensaje implícito en el comentario)

Separación entre los roles adjudicados a la mujer y al hombre.

\section{Cambiando} el Discurso (deconstrucción)

\section{El hombre y la} mujer tienen los mismos derechos y responsabilidades ante la crianza de los menores.

Ella es la que está mal en las áreas cognitiva, afectiva y conductual. Ella tiene que siempre aceptar mis opiniones o mandatos.

Responsabiliza a ella por sus actos violentos.

Reconocer, aceptar y validar opiniones, ideas, sentimientos y las propias.

\section{Cada cual es} responsable de sus conductas diferentes a propios actos. Todos tienen la responsabilidad de aprender a manejar adecuadamente el coraje para evitar llegar a la ira.

\section{Minimiza la conducta violenta.}

La mujer es responsable de los deberes del hogar.

\section{El hombre se siente} superior a la mujer en toma de decisiones y en sus acciones.

\section{Las amenazas} constituyen violencia en la relación de pareja.

Las tareas del hogar no son exclusivas de un sexo.

La mujer es capaz de tomar decisiones responsablemente y estar consciente de las consecuencias de sus acciones. 
De esta forma, a través de la metodología grupal, los agresores son expuestos, entre otras cosas, a una diversidad de temas. Además, se discuten las narraciones propias de estos y se trabaja con los discursos presentados por los mismos, de tal manera que logren cambios favorables en sus áreas cognitivas, afectivas y conductuales.

\section{Conclusión}

El trabajo de intervención profesional a nivel de prevención primaria y secundaria que se ha estado realizando con los agresores y las agresoras de violencia doméstica está a penas en sus inicios. Se espera que tanto profesionales del trabajo social como otros profesionales de la conducta humana le presten atención específica a esta población a fin de que los agresores y las agresoras logren modificar y cambiar aspectos conductuales, cognoscitivos y afectivos. Esta población no puede seguir siendo invisible ante los ojos de los profesionales de la conducta humana. 


\section{Referencias}

Arrigoni, Flavia, Jiménez, José Luis, Navarro, Josél., \& Mendoza, Pedro (2013). Aplicación de un programa terapéutico en hombres violentos contra la pareja. Anuario de Psicología Jurídica 2013. 23, 3-9. doi: http://dx.doi.org/10.5093/ aj2013a2.

Bandura, Albert (1986). Social Foundations of Thought and Action. Englewood Cliffs: Practice Hall.

Corsi, Jorge, Dohmen, Mónica Liliana, \& Sotés, Miguel Ángel (2004). Violencia masculina en la pareja. México: Paidós.

Decker, David J. (1999). Stopping the violence: A group model to change men's abusive attitudes and behaviors. New York: The Haworth Maltreatment and Trauma Press.

Ley para la Prevención e Intervención con la Violencia Doméstica, Ley Núm. 54 de 1989, 8 L.P.R.A. § 601 et seq.

Molina-Castro, Gloria M. (2006). La prevención y la metodología grupal como elementos importantes para una práctica efectiva en trabajo social. Revista ANÁLISIS, VII (1), 1-13.

Molina-Castro, Gloria M., \& Fernández, Antonio (2009). Perfil del agresor y algunos procesos de la intervención grupal. Scientific International Journal, 6 (1), 15-37.

Molina-Castro, Gloria M., Fernández, Antonio, \& Berríos, Yarisel (2015). Efectividad de los Servicios Socio-Educativos a Agresores de Violencia Doméstica a través del Proyecto de Resocialización Masculina y Femenina. Scientific International Journal, 12 (2), 5-18.

Ortiz, Alejandrina, \& García, Emilio (2004). Violencia Doméstica: Modelos de análisis de programas de intervención con agresores. Revista Jurídica de LexJuris. Recuperado de: 
http://www.lexjuris.com/revista/opcion1/2003Violencia\%20 Domestica-\%20Modelo\%20de\%20Analisis\%20y\%20 Programas.htm.

Pérez, Natalie (2011). La otra cara... Intervención Grupal con el agresor de violencia doméstica y algunas críticas hacia la ley de aplicabilidad. Scientific International Journal, 8 (2), 37.

Pransky, Jack (2003). Prevention from the inside-out. Springfield: Burrell Foundation \& Paradigm Press. 